\title{
Zur eidgenössischen Volksabstimmung vom 19./20. Mai 1973 über die Aufhebung der Ausnahmeartikel 51 und 52 der Bundesverfassung
}

Werner Mettler

Die Stimmberechtigten der Schweiz hatten am 19./20. Mai 1973 über die Aufhebung der Artikel 51 und 52 der Bundesverfassung (BV) zu befinden. Der Artikel 51 der BV verbot den Jesuiten die Tätigkeit in Kirche und Schule. In Artikel 52 der BV wurde die Errichtung neuer oder die Wiederherstellung aufgehobener Klöster oder religiöser Orden untersagt. Die Abstimmung bewies, bei einer knapp über dem Durchschnitt (40\%) liegenden Beteiligung, daß, wiewohl mit einer ebenfalls schwachen Mehrheit von 55\%, die Ansicht überwog, die Artikel seien überholt. Dieser Entscheid dürfte gewiß die künftige konfessionelle Verständigung fördern. Für den Geographen stellt sich hieraus die Frage, ob von jenem auch geographische Konsequenzen zu erwarten seien.

Um hierauf antworten zu können, ist zunächst das räumliche Gefüge der Abstimmung selbst zu untersuchen. Es zeigt in der Tat erhebliche Unterschiede, die in den folgenden Karten festgehalten sind:

Die Karte 1 stellt die räumliche Verteilung der JaStimmen dar. Mit einer Mehrheit von über 75\% Ja-Stimmen haben die zehn Kantone Appenzell Innerrhoden, Freiburg, Luzern, Nidwalden, Obwalden, Schwyz, Tessin, Uri, Wallis und Zug die Vorlage angenommen. In diesen vorwiegend katholischen Ständen waren die Klöster begreiflicherweise bis in die Gegenwart so gut wie unbehelligt geblieben, was nicht zuletzt mit dem Intaktbleiben der religiösen Institutionen zusammenhängt, welche noch heute vielfach die Schulung (Kollegien) und die Fürsorge tragen. Auch in den gemischtkonfessionellen Kantonen Graubünden, Solothurn und St. Gallen unterstützte eine deutliche Mehrheit die Vorlage. Während für Solothurn, das noch heute landschaftlich sehr stark durch die Klöster geprägt wird, die Interpretation verständlich ist, liegen die Motive bei Graubünden kaum in der konfessionellen Entwicklung. In den knapp annehmenden Kantonen Aargau, Basel-Landschaft, Basel-Stadt, Genf, Glarus und Thurgau ist wohl die mit der starken Industrialisierung zusammenhängende Bevölkerungsdurchmischung der Hauptgrund für das genannte Abstimmungsergebnis. Bemerkenswert hoch ist die Anzahl der die Vorlage ablehnenden Kantone Appenzell Außerrhoden, Bern, Neuenburg, Schaffhausen, Waadt und Zürich. Unter ihnen weist der Kanton Bern den zweitgrößten prozentualen Anteil an Nein-Stimmen auf, obwohl er eine erhebliche Mehrheit an Ja-Stimmen im katholischen Jura besitzt.

Die Karte 2 orientiert über die Stimmenbeteiligung, die in allen Kantonen in den traditionellen Grenzen lag.

In Karte 3 wird die konfessionelle Verteilung der Bevölkerung nach Kantonen dargestellt.

Der Anteil der Ja-Stimmen (54.9\%) stimmt - begreiflicherweise - mit dem Anteil der katholischen Bevölkerung (52,3\%) weitgehend überein. Auch die Karte 1 weist eine - zwar zu erwartende, aber dennoch bemerkenswerte - Ahnlichkeit mit der Karte 3 auf. Deshalb soll mit Hilfe des Korrelationskoeffizienten, der ein $\mathrm{Maß}$ für die lineare $\mathrm{Ab}$ hängigkeit ist, und der Regressionsgleichung, welche die Funktionsgerade für die Variable $Y$ bezüglich der Variabeln A angibt, das Phänomen untersucht werden.

$$
\begin{aligned}
& \text { Ansatz: } Y=f(X) \text { wobei } \\
& \mathrm{X}=\text { Anteil der Katholiken in Prozent } \\
& \mathrm{Y}=\mathrm{Ja} \text {-Stimmen in Prozent } \\
& f(X)=\text { lineare Funktion von } X \text {; } \\
& \text { z. B. } Y=A_{0}+A_{1} \cdot X
\end{aligned}
$$

Die Abbildung 4 zeigt deutlich die lineare Abhängigkeit der Variablen Y von der Varibalen A; sie ist bei einem Korrelationskoeffizienten von 0.959 hochsignifikant.

Gegen diese Schlüsse lassen sich verschiedene Einwände erheben. Vor allem kann gesagt werden, die Untersuchung beschränke sich ausschließlich auf den Vergleich Konfession/Stimmenzahl, und dazu seien die Nichtstimmbeteiligten miteinbezogen. Die zur Verfügung stehenden Unterlagen geben aber keinen Hinweis auf den Anteil der stimmberechtigten Katholiken, ganz abgesehen von denjenigen, die auch zur Urne gingen. Deshalb wurde der gesamte prozentuale Anteil Katholiken an der Bevölkerung berücksichtigt. Andere, das Abstimmungsresultat beeinflussende Faktoren wie die parteipolitischen Strukturen schienen weniger entscheidend gewesen zu sein. Nicht berücksichtigt werden konnten die sehr schwer zu ermittelnden Größen wie die Erfahrung der Bevölkerung mit den Jesuiten, oder die Beziehung zwischen den Klöstern und ihrer Umwelt, oder schließlich der Grad der ökumenischen Gemeinschaften in den Gemeinden und Kantonen. 


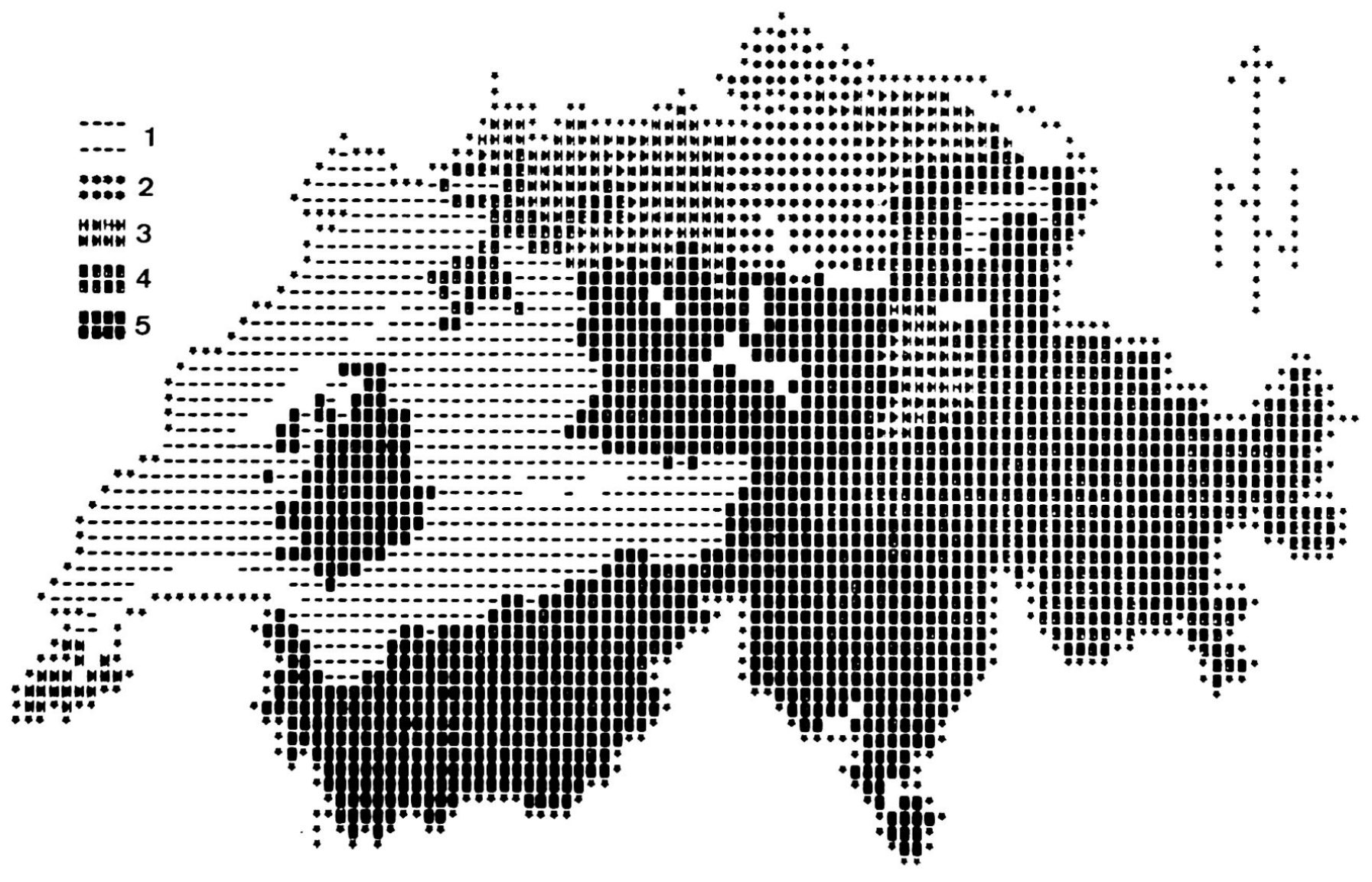

Karte 1. Prozentualer Anteil der Ja-Stimmen, gegliedert nach Kantonen

1 25-40\% Ja-Stimmen, 2 40-50\% Ja-Stimmen, 3 50-60\% Ja-Stimmen, 4 60-75\% Ja-Stimmen,

5 75-95\% Ja-Stimmen

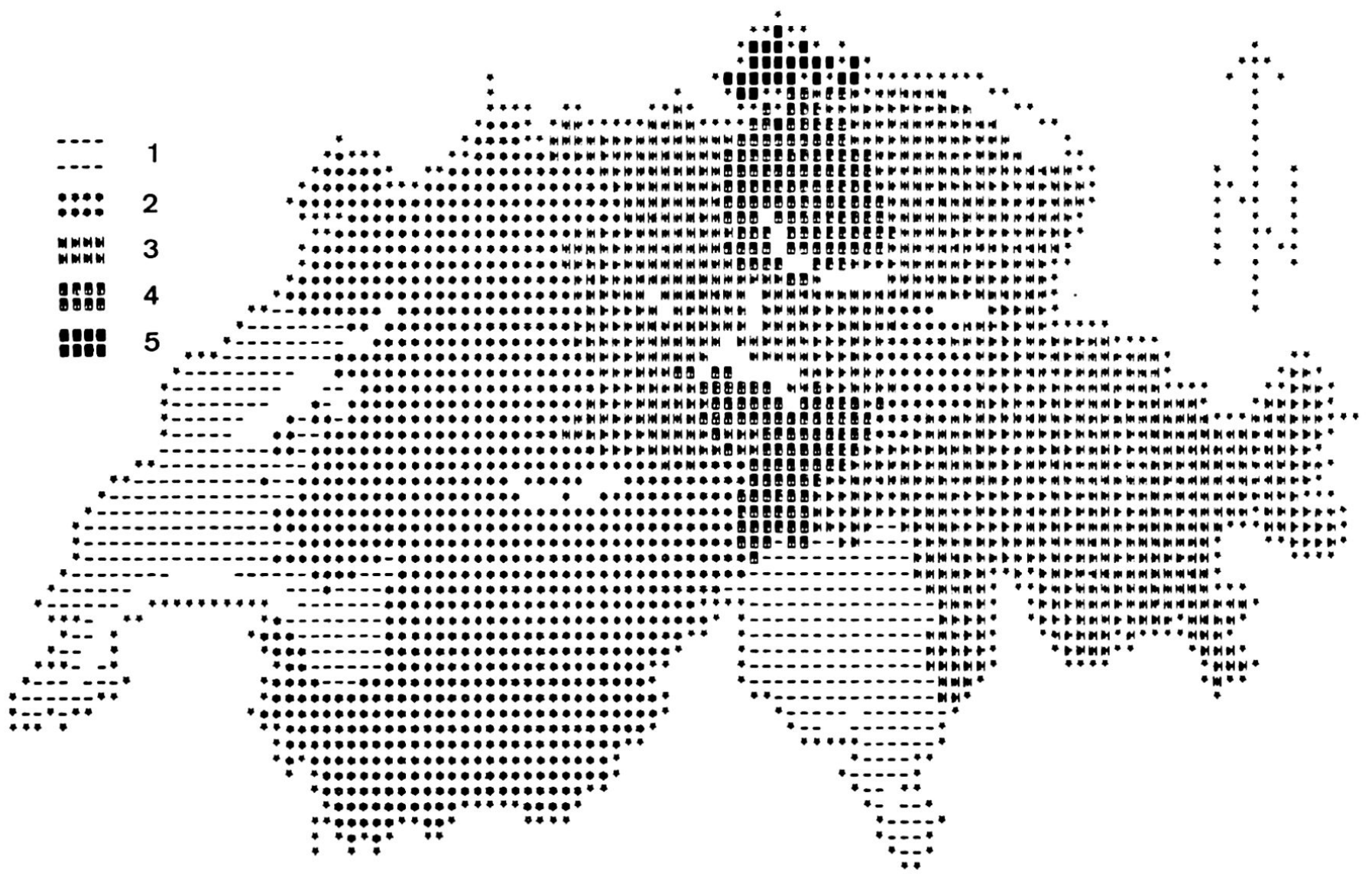

Karte 2. Stimmbeteiligung in Prozent, geliedert nach Kantonen

$120-30 \%$ Stimmbeteiligung, $2 \quad 30-40 \%$ Stimmbeteiligung, $3 \quad 40-50 \%$ Stimmbeteiligung. $4 \quad 50-60 \%$ Stimmbeteiligung, 5 60-70\% Stimmbeteiligung 


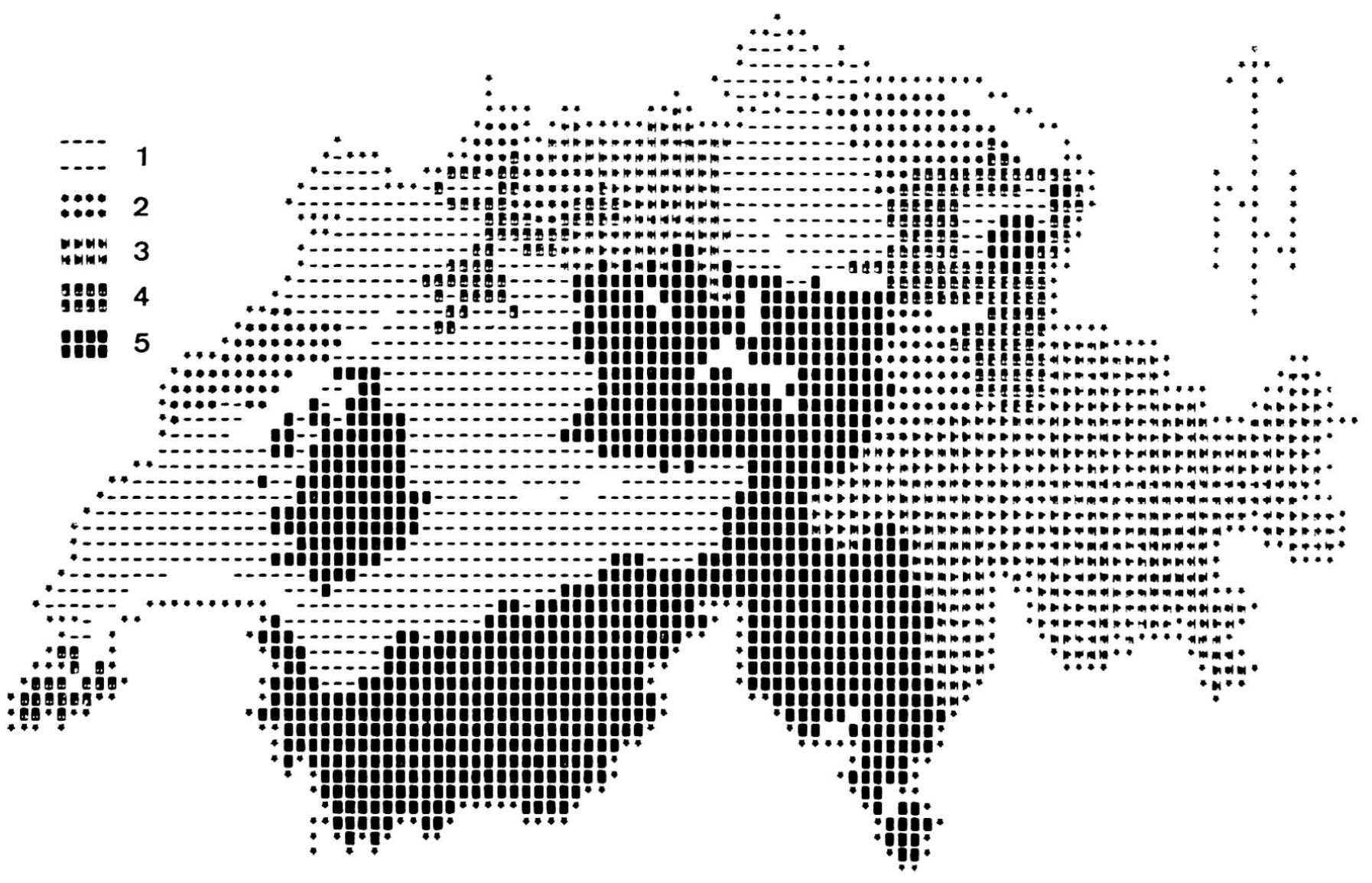

Karte 3. Prozentualer Anteil der katholischen Bevölkerung an der Gesamtbevölkerung, gegliedert nach Kantonen. $120-40 \%$ Katholiken, $240-50 \%$ Katholiken, 3 50-60\% Katholiken, 4 60-80\% Katholiken, $580-100 \%$ Katholiken

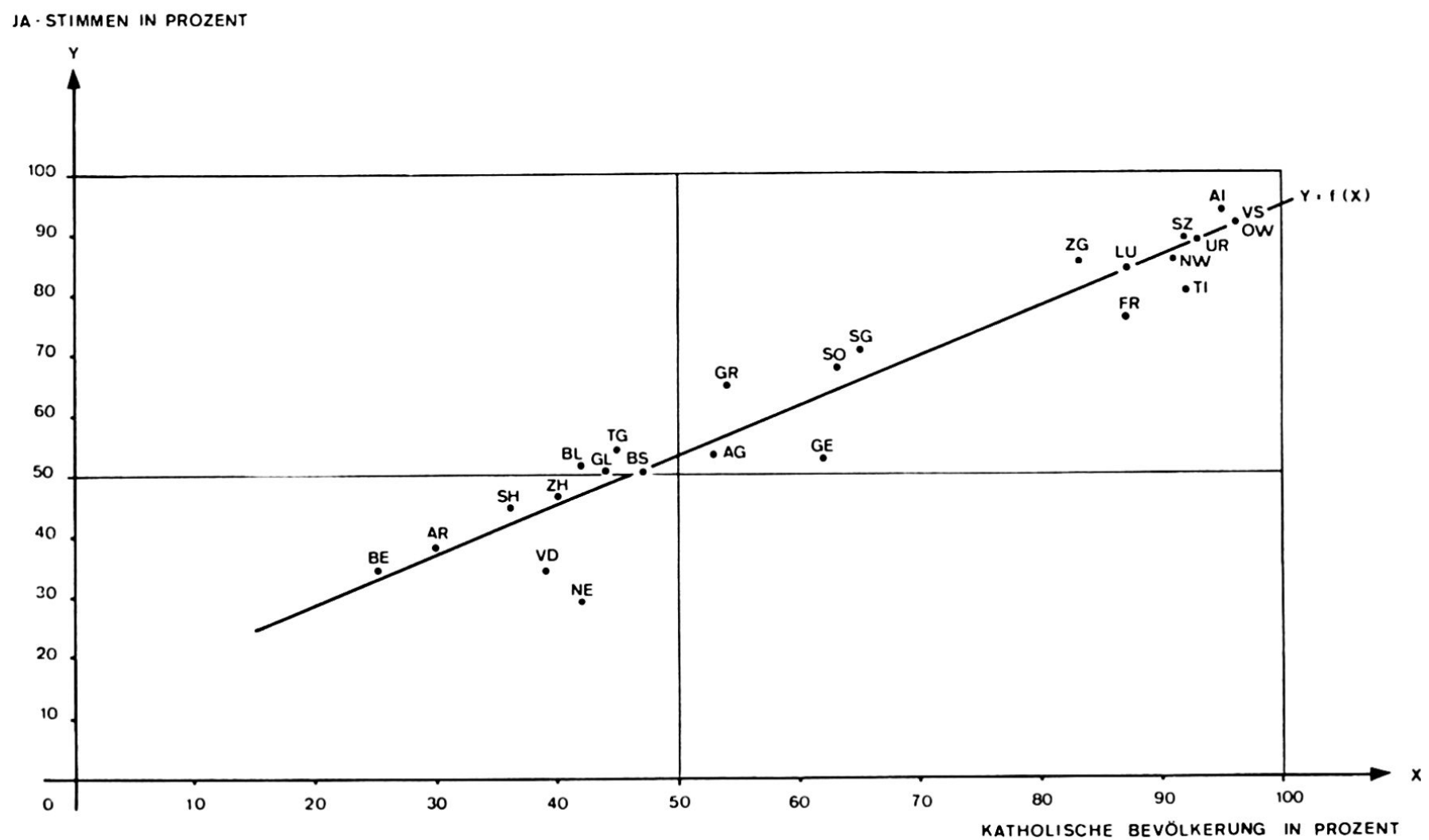

Abbildung 4. Vergleich der Variablen X, prozentualer Anteil der Katholiken, mit der Variablen Y, prozentualer Anteil der Ja-Stimmen. Die Regressionsgerade $\mathrm{Y}=\mathrm{f}(\mathrm{A})$ hat die Form $\mathrm{Y}=12,08+0,82 \mathrm{X}$ 
Das eigentlich geographische Problem allerdings, die Frage nach den zu erwartenden landschaftlichen Konsequenzen der Abstimmung aufgrund der genannten Daten ist kaum konkret und vor allem nicht unmittelbar zu lösen. Mit der Aufhebung der Ausnahmeartikel haben Jesuiten wie Klöster erneut "normale» Rechte erlangt. Sie wecken damit die Hoffnung, daß sie auch künftig im Interesse harmonischer, gesunder - insbesondere natürlich schweizerischer - Kulturlandschaftsentwicklung wirken werden. Vor allem können dabei die Klöster auf eine lange diesbezügliche praktische Arbeit hinweisen. Wenn auch nicht vorauszusehen ist, daß sie sich in Zukunft vermehren werden, besteht die
Möglichkeit der landschaftlichen Einflußnahme prophylaktisch wie konservierend und aktiv (therapeutisch) - durchaus. Äußerungen zuständiger Persönlichkeiten zufolge ist diese Aufgabe zweifellos erkannt worden.

Adresse des Verfassers:

Werner Mettler

Buchthalerstraße 127, 8203 Schaffhausen

Geographisches Institut ETHZ

Sonneggstraße 5, 8006 Zürich 